\title{
Measurement of Coracohumeral Distance: A New Method Using a 3-Dimensional Multiplanar Reformatted MR Image
}

\author{
Hye Jin Yoo ${ }^{1}$, Hye Yeon $\mathrm{Oh}^{1}$, Sung Hwan Hong ${ }^{1}$, Ja Young Choi ${ }^{1}$, Hee Dong Chae ${ }^{1}$ and Sae Hoon Kim*2 \\ ${ }^{1}$ Department of Radiology, Seoul National University College of Medicine, Korea
}

${ }^{2}$ Department of Orthopedic Surgery, Seoul National University College of Medicine, Korea

Received: 制: October 01, 2018; Published: iㅔ: October 11, 2018

*Corresponding author: Sae Hoon Kim, Department of Orthopedic Surgery, Seoul National University College of Medicine, Korea

\begin{abstract}
Objective: The purpose our study was to demonstrate a new method to reliably measure the coracohumeral distance (CHD) using a 3-dimensional multiplanar reformatted (3-D MPR) MR image.

Materials and Methods: Seventy-seven shoulder MRIs were retrospectively reviewed (32 males, 45 females; $61.6 \pm 6.6$ years). The patients were categorized based on the extent of the subscapularis tendon (SScT) tear: normal tendon (SScT0) (n=32); full-thickness tear involving $<70 \%$ of tendon SScTF) ( $n=38$ ); near-complete tear involving $\geq 70 \%$ of tendon (SScTC)( $n=7)$. Twenty-five patients were also analyzed as a control group. The shortest CHD was measured on both traditional axial images (CHDax) and MPR MR images (CHD3D) using a new method. The correlation between CHDax and CHD3D was evaluated. Then, differences in CHD among groups were analyzed. Several MR imaging findings were assessed to determine whether they had any relationship with CHDs.
\end{abstract}

Results: There was almost perfect agreement between the readers in measuring CHDs. There was a strong correlation of 0.77 ( $p<0.001$ ) between CHDax and CHD3D values. For SScT abnormality, the CHD3D was significantly narrowed in the SScTC group, compared to the control and other patient groups $(\mathrm{p}=0.049)$. However, there was no correlation between the CHDax and SScT abnormalities $(\mathrm{p}=0.401)$. Lesions of the long head of the biceps tendon and decentering of the humeral head were significantly associated with a SScT tear $(\mathrm{p}<0.001)$.

Conclusion: CHD3D could be reliably measured on MPR images using the new method. The CHD3D was smaller in patients with a near complete tear of the SScT, compared to those without. There was a greater statistical correlation of SScT abnormalities with CHD3D than with traditional CHDax.

Keywords: Coracohumeral Distance; Subscapularis Tendon Tear; Subcoracoid Impingement; Magnetic Resonance Image; Multiplanar Reformatted Image; 3-Dimensional

\section{Introduction}

Subcoracoid impingement syndrome is known as an unusual cause of anterior shoulder pain [1-8]. However, it remains a poorly understood disease entity and it is still difficult to diagnosed accurately with both physical examination and imaging studies $[2,5]$. Clinically, this syndrome is characterized by anterior shoulder pain that is aggravated by adduction, internal rotation, and forward flexion and may be relieved by diagnostic injection $[2,4,5,9]$. The pain could be provoked in this position by the entrapment of soft tissues including the subscapularis tendon, middle glenohumeral ligament, and long head of biceps tendon between the coracoid process and the humeral head $[2,4,5,9]$. Therefore, many investigators have thought that subcoracoid impingement syndrome may occur in association with subcoracoid stenosis. The coracohumeral distance (CHD) has been widely evaluated in both healthy volunteers and patients with subcoracoid impingement syndrome because it could be a parameter that reflect the degree of subcoracoid stenosis [2,4,5,8-12]. However, the CHD has been shown to be a poor predictor of subcoracoid impingement on imaging studies $[4,5,8,9,13,14]$.

One possible reason for this debatable result may be the limitations of imaging studies acquired with a fixed specific position and orientation of the sectional plane. Most CHDs in previous studies was measured on a two-dimensional (2-D) image plane such as an axial or an oblique sagittal image plane. However, these 2-D images usually do not include the true center of rotation of the shoulder, therefore the distance measured on a 2-D image is not the true shortest distance between the coracoid process and the lesser tuberosity of the humerus and may be seriously 
compromised by projectional artifacts. Thus, it may not truly reflect subcoracoid stenosis. Several previous studies have shown that 2-D imaging was also problematic in the measurement of the humero-acromial distance in subacromial impingement patients, but this limitation can be overcome by acquiring complete 3-D data sets of the shoulder and measuring the true minimal 3-D distance between the humerus and the acromion after 3D reconstruction of these structures $[2,15,16]$. However, there has been no previous investigation to measure the true shortest CHD independent of the sectional orientation using a 3-D reconstruction technique.

Therefore, the purpose of our study was to determine whether the true shortest 3-D distance between the coracoid process and the lesser tuberosity of the humerus can be reliably measured with a new method using 3-dimensional multiplanar reformatted (3-D MPR) MR image.

\section{Materials and Methods}

\section{Patient}

Our institutional review board approved this retrospective study. Between August 2013 and December 2014, 192 consecutive patients treated with arthroscopic rotator cuff repair were initially included in the patient group of this study. Among them, 122 patients were excluded because there was no preoperative shoulder MRI $(n=10)$, or because the MRI was performed at an outside hospital (n=112). Finally, 77 patients with preoperative shoulder MRIs performed at our hospital were included in this study as the patient group (mean age \pm standard deviation, $61.6 \pm 6.6$ years; age range, 43-77; Male/Female, 32/45; Right/Left, 54/23). The extent of the rotator cuff tendon tears was confirmed by arthroscopic surgery. All patients had full thickness tears in the supraspinatus tendon (SST). The length of subscapularis tendon (SScT) tear was described as the superior-to-inferior length of the tendinous portion, excluding the muscular portion which corresponded to $1 / 3$ of the SScT insertion that had torn away from its insertion on the lesser tuberosity and this length was converted to a percentage of tendon attachment that was torn [12]. Involvement of the muscular insertion in a SScT tear is very rare and usually muscular portion is preserved in an anterosuperior rotator cuff tear $[17,18]$. The patients were categorized based on the extent of the SScT tear: normal tendon (SScT0) (n=32); full thickness tear less than $70 \%$ of tendon attachment (SScTF) ( $n=38)$; near complete tear having a full thickness tear equal to or greater than $70 \%$ of tendon attachment (SScTC) ( $n=7)$. Twenty-five control group patients were selected with the criteria of having nondiagnosis of any impingement syndrome or no anterior shoulder pain and they were analyzed against the patient group (age range, 32-75; mean age \pm standard deviation, 53.1 \pm 10.6 years; Female/Male, 19/6; Right/Left, 10/15). The control group patients had been referred for an MRI with the suspicion of other shoulder-related problems, such as labral or glenohumeral ligament tears, bursitis, adhesive capsulitis, etc.

\section{MR Imaging}

All the shoulder MRI exams were performed in our hospital using 1.5T (SignaHDxt 1.5T, GE Healthcare, Milwaukee, WI) or 3.0 T (MAGNETOM Trio/Verio, Siemens Medical Solutions,
Erlangen, Germany) MR scanners with a dedicated shoulder coil. The patients were placed in the supine position with the forearm turned to the semi-pronation (neutral) position. The MR imaging protocol included the following sequences: axial fast spin-echo proton-density weighted image with fat saturation (TR/TE, 23003900/30-60 ; slice thickness, $3 \mathrm{~mm}$; slice gap, $0.3 \mathrm{~mm}$; field of view, $14 \mathrm{~cm}$, matrix, 384x269; and echo train length 7); oblique coronal and sagittal fast spin- echo T2-weighted images with fat saturation (TR/TE,2300-4600/30-50; slice thickness, 3mm; slice gap, 0mm; field of view, $14 \mathrm{~cm}$, matrix, 384x269; and echo train length 12 ); and oblique coronal and extended oblique sagittal fast spin-echo T1-weighted images (TR/TE, 400-800/10-15ms; slice thickness, $3-4 \mathrm{~mm}$; slice gap, $0-0.4 \mathrm{~mm}$; field of view, $14 \mathrm{~cm}$ for coronal, $16 \mathrm{~cm}$ for sagittal images; matrix, 384x269; and ETL 4). Extended oblique sagittal T1-weighted images were taken with larger field of view $(16 \mathrm{~cm})$ covering the inferior border of the scapula body to obtain the far medial section of rotator cuff muscles.

\section{Generation of Multiplanar Reformatted (MPR) MR images and Measurement of CHD}

The minimal coracohumeral distance (CHD) was measured on both traditional axial image (CHDax) and multiplanar reformatted (MPR) images (CHD3D). The MPR image (CHD3D) was obtained by following the new MPR method: First, axial MR images were transferred to a PACS workstation (INFINITT, Infinitt healthcare, Seoul, Korea), and MPR images in the standard axial and coronal planes were generated using a 3-D reconstruction function of our PACS system (Xelis 3D, Infinitt healthcare, Seoul, Korea). Second, the axial center of the humeral head was determined on an axial MPR image by placing the best-fit circle along the articular surface of the humeral head (Figure 1a). Third, coronal oblique MPR images were generated by adjusting the coronal image axis to pass through the axial center of the humeral head and perpendicular to the glenoid surface (Figure 1b). Fourth, the 3-D center of rotation (COR) of the humeral head was determined on a coronal oblique MPR image by placing the best-fit circle along the articular surface of the humeral head, ensuring that the center of the circle overlaid the sagittal axis line (Figure 1c). Fifth, the axial image axis on a coronal oblique MPR image was moved to pass through the 3-D COR of the humeral head (Figure 1d). Sixth, the shortest distance between the coracoid process and the 3-D COR of the humeral head $(\mathrm{Co}-\mathrm{H})$ was defined by adjusting the sagittal axis on an axial image (Figure 1e) and the axial axis on a sagittal image (Figure 1f), so that both image axes passed through the 3-D RC of the humeral head and the coracoid process. Seventh, the shortest distance between the lesser tuberosity cortical margin and the 3-D COR of the humeral head (LT-H) was defined by adjusting the sagittal axis on an axial image (Figure 1g) and the axial axis on a sagittal image (Figure 1h), so that both image axes passed through the 3-D RC of the humeral head and the lesser tuberosity of humeral head. Finally, the CHD 3D was calculated by subtracting the LT-H from the C-H. To test the interobserver agreement in measurement of the CHD, two fellowship-trained radiologists, with 8 and 5 years of experience in musculoskeletal MR interpretation, independently measured the CHDax and CHD3D. 


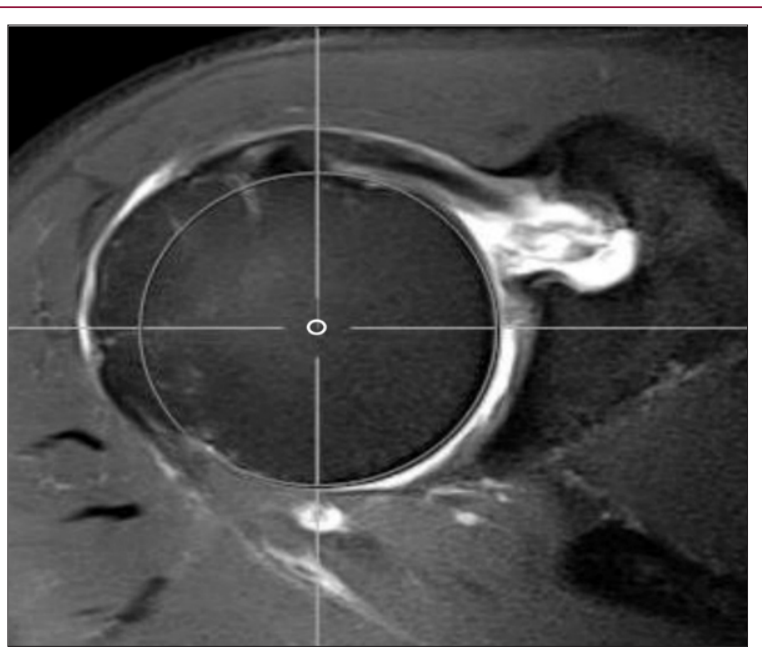

Figure 1a: A new method to determine the minimal coracohumeral distance (CHD) using MPR MR image.

a: Orthogonal axial Proton-density (PD) MR image with fat-saturation. The axial center (open circle) of the humeral head was determined on an axial image by placing the best-fit circle along the articular surface of the humeral head.

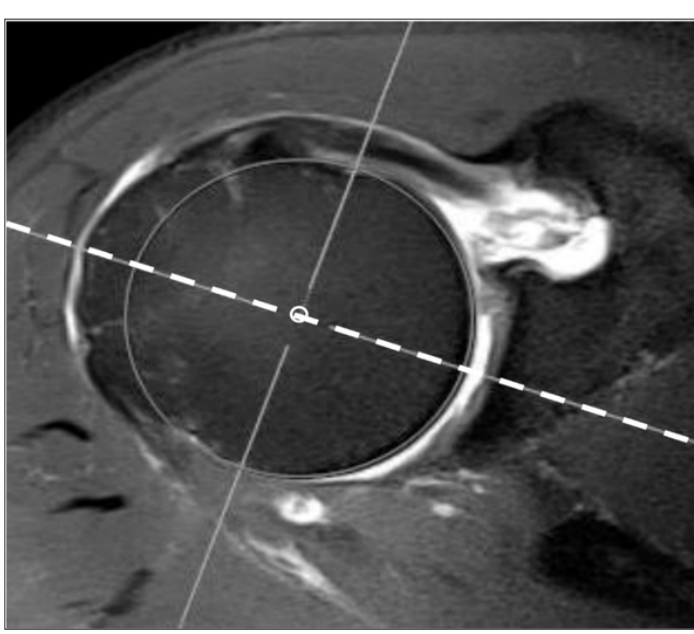

Figure 1b: A new method to determine the minimal coracohumeral distance (CHD) using MPR MR image.

b: Orthogonal axial Proton-density (PD) MR image with fat-saturation. Coronal oblique MPR images were generated by adjusting the coronal image axis (dashed line) to pass through the axial center (open circle) of the humeral head and perpendicular to the glenoid surface.

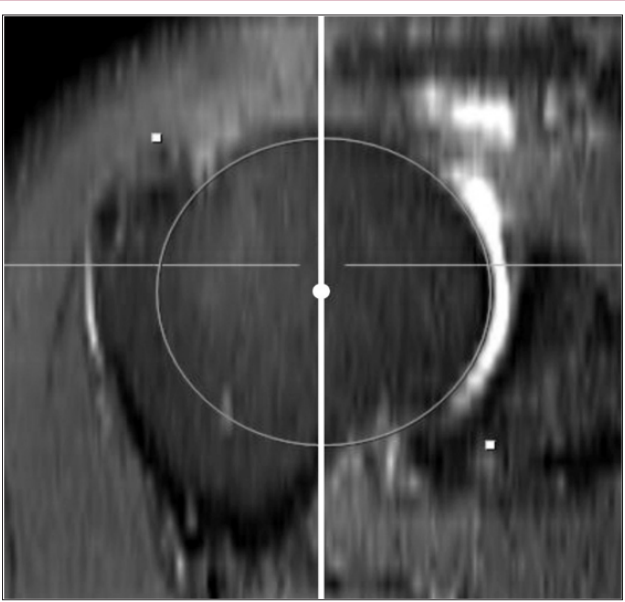

Figure 1c: A new method to determine the minimal coracohumeral distance (CHD) using MPR MR image.

c: Coronal oblique MPR MR image generated from figure 1b. The 3-D center of rotation (COR) of the humeral head (circle) was determined on coronal oblique MPR images by placing the best-fit circle along the articular surface of the humeral head, ensuring that the center of the circle overlaid the sagittal axis line (vertical solid line). 


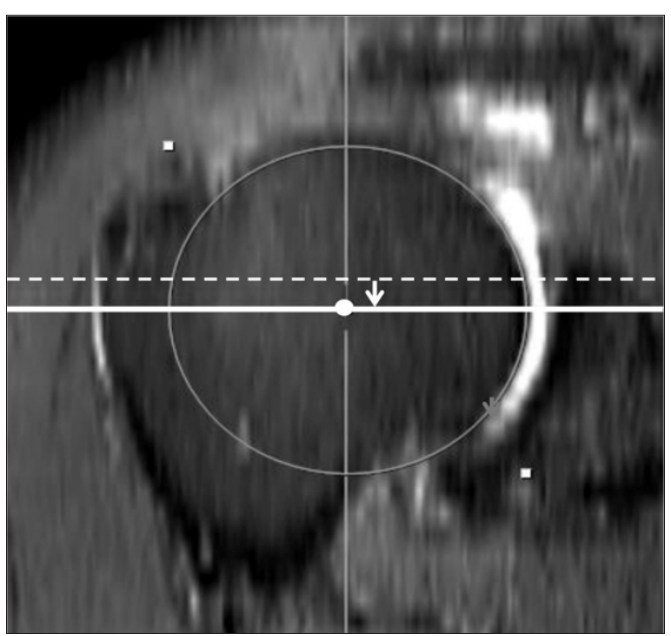

Figure 1d: A new method to determine the minimal coracohumeral distance (CHD) using MPR MR image.

$\mathrm{d}$ : Coronal oblique MPR MR image generated from fig $1 \mathrm{~b}$. The axial axis (from dashed line to solid line) in a coronal oblique MPR image was moved to pass through the 3-D COR of the humeral head (circle).

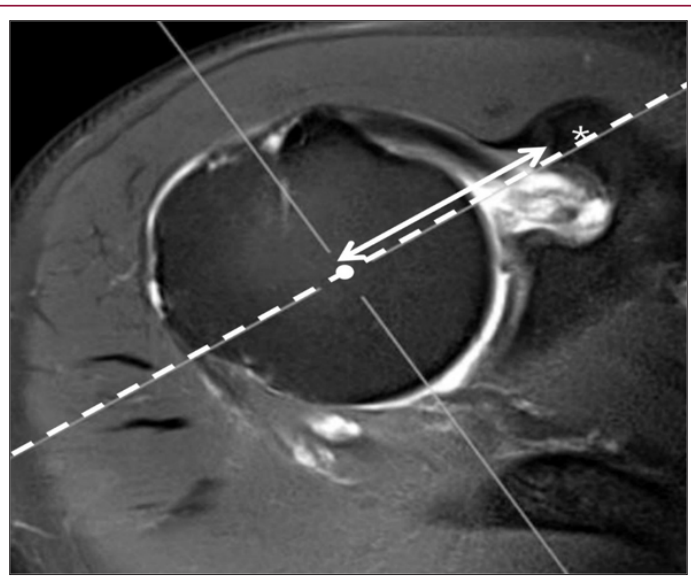

Figure 1e: A new method to determine the minimal coracohumeral distance (CHD) using MPR MR image.

e: Measurement of the distance between the coracoid process and the COR of the humeral head $(\mathrm{Co}-\mathrm{H})$ on axial and sagittal MPR MR images generated from figure $1 \mathrm{~d}$. The shortest distance between the coracoid process $\left(^{*}\right)$ and the 3-D COR of the humeral head $(\mathrm{Co}-\mathrm{H}$, left right arrow) was determined by adjusting the sagittal axis (dashed line on figure 1e) in an axial MPR image and axial axis (dotted line on figure 1f) in a sagittal MPR image, so that both image axes pass through the 3-D COR of the humeral head and coracoid process $\left(^{*}\right)$.

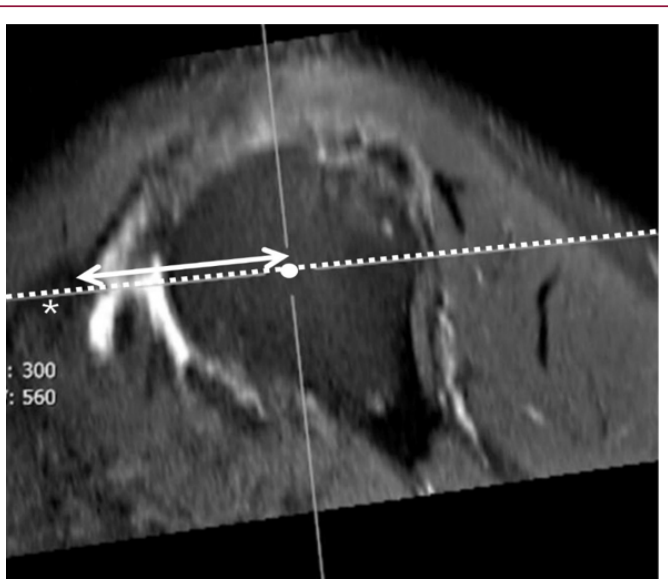

Figure 1f: A new method to determine the minimal coracohumeral distance (CHD) using MPR MR image.

$\mathrm{f}$ : Measurement of the distance between the coracoid process and the COR of the humeral head $(\mathrm{Co}-\mathrm{H})$ on axial and sagittal MPR MR images generated from figure $1 \mathrm{~d}$. The shortest distance between the coracoid process $\left(^{*}\right)$ and the 3-D COR of the humeral head $(\mathrm{Co}-\mathrm{H}$, left right arrow) was determined by adjusting the sagittal axis (dashed line on figure 1e) in an axial MPR image and axial axis (dotted line on figure 1f) in a sagittal MPR image, so that both image axes pass through the 3-D COR of the humeral head and coracoid process $\left(^{*}\right)$. 


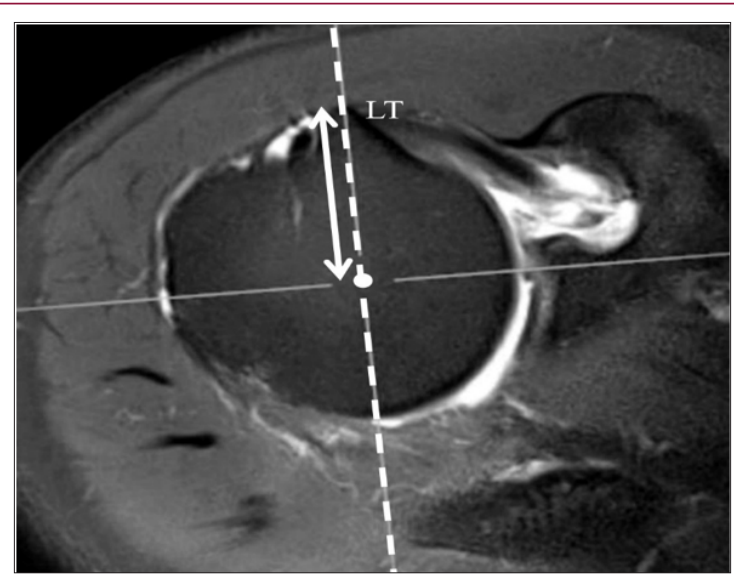

Figure 1g: A new method to determine the minimal coracohumeral distance (CHD) using MPR MR image.

g: Measurement of the distance between the lesser tuberosity of the humeral head and the COR of the humeral head (LT-H) on axial and sagittal MPR MR images generated from fig 1d. The shortest distance between the lesser tuberosity (LT) and the 3-D COR of humeral head (LT-H, up down arrow) was determined by adjusting the sagittal axis (dashed line on fig $1 \mathrm{~g}$ ) in an axial image and the axial axis (dotted line on fig $1 \mathrm{~h}$ ) in a sagittal image, so that both image axes pass through the 3-D COR of humeral head (white circle) and lesser tuberosity (LT).

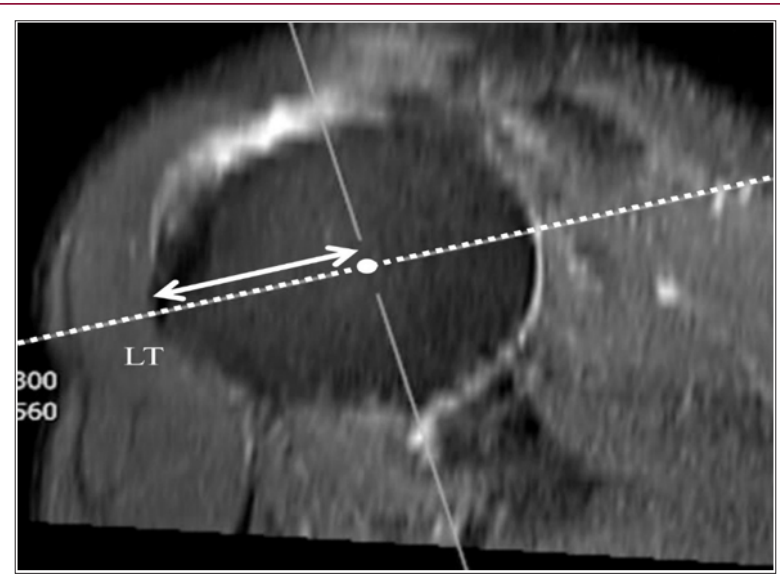

Figure 1h: A new method to determine the minimal coracohumeral distance (CHD) using MPR MR image.

h: Measurement of the distance between the lesser tuberosity of the humeral head and the COR of the humeral head (LT-H) on axial and sagittal MPR MR images generated from fig 1d. The shortest distance between the lesser tuberosity (LT) and the 3-D COR of humeral head (LT-H, up down arrow) was determined by adjusting the sagittal axis (dashed line on fig $1 \mathrm{~g}$ ) in an axial image and the axial axis (dotted line on fig $1 \mathrm{~h}$ ) in a sagittal image, so that both image axes pass through the 3-D COR of humeral head (white circle) and lesser tuberosity (LT).

\section{Image Analysis}

In each group, the following imaging findings were evaluated to assess their relationship with a SScT abnormality: subcoracoid bursa, long head of biceps tendon (LHBT) abnormality, subcortical bone change in the lesser tuberosity of the humeral head, and Decentering of humeral head within the glenoid fossa [19]. Lesions of the LHBT were classified as to whether the tendon continuously existed and then classified as to whether it was unstable. First, if the continuity of the tendon was not preserved, it was classified as a complete tear. Second, when the biceps tendon was subluxated or dislocated, the tendon was defined as unstable. Third, the other biceps pathologies including degenerative change and partial tear were classified as stable LHBTs. Subcortical bone changes of the LT included subcortical bone marrow (BM) edema and cystic change. Decentering of the humeral head was the state in which the center of humeral head translated from a centerline obtained perpendicular to the glenoid surface.

\section{Statistical Analysis}

Interobserver agreement of the CHD measurement was assessed with intraclass correlation coefficients (ICCs) and by using a two-way random average measures model with consistency type [19-21]. In general, agreement was defined as poor $(<0.0)$, slight (0.0-0.2), fair (0.21-0.4), moderate (0.41-0.6), substantial (0.61$0.8)$, or almost perfect (0.81-1.0). A correlation analysis between the CHDax and CHD3Dwas performed using Pearson's correlation coefficient. Differences in CHD between groups were analyzed with ANOVA (Analysis of Variance). A p-value $<0.05$ was considered significant. The other nonparametric variables were processed by chi-square analysis or Fisher's exact test. The statistical analyses were performed using SPSS version 21.0 (SPSS Inc., Chicago, IL, USA) and MedCalc version 12.7.7 (MedCalc Software, Ostend, Belgium). 


\section{Results}

There was almost perfect agreement between the readers in measuring both the CHDax (ICC, 0.947) and CHD3D (ICC, 0.868). There was a good correlation between the CHDax and CHD3D (Pearson's correlation coefficient $=0.77, \mathrm{p}<0.001$ ).

The CHDs in the control and patient groups are summarized in Table 1 . The ANOVA showed that there was a statistically significant difference in the CHD3D among the groups ( $p=0.049)$. Post hoc tests demonstrated that the CHD3D was significantly narrowed in the group of patients with a near-complete SScT tear (SScTC), compared to the group of patients with a normal SScT (SScT0, $\mathrm{p}=0.050$ ) and the group of patients with $\mathrm{a}<70 \%$ full-thickness tear in the SScT (SScTF, p=0.034). In the patients with a normal SScT, a supraspinatus tendon tear did not affect the $\mathrm{CH}$ narrowing (control vs. SScT0, p=0.985). However, the present study failed to show a significant difference in CHDax among the control and patient groups. Even in the SScTC group, the CHDax was not different from that of the control group $(\mathrm{p}=0.401)$.

Table 1: Coracohumeral distance on axial image (CHDax) and MPR image (CDH3D).

\begin{tabular}{|c|c|c|c|}
\hline & Number of patients & CHD $_{\text {ax }}$ & CDH $_{3 \mathbf{D}}$ \\
\hline Control & 25 & $9.49 \pm 1.42$ & $4.25 \pm 1.88$ \\
\hline${ }^{1} \mathrm{SScT}_{0}$ & 32 & $9.50 \pm 2.75$ & $4.48 \pm 2.48$ \\
\hline${ }^{2} \mathrm{SScT}_{\mathrm{F}}$ & 38 & $9.46 \pm 2.71$ & $4.62 \pm 2.60$ \\
\hline${ }^{3} \mathrm{SScT}_{\mathrm{C}}$ & 7 & $7.78 \pm 3.42$ & $1.81 \pm 3.54$ \\
\hline$p$ value & & 0.401 & $0.049^{*}$ \\
\hline
\end{tabular}

Data $(\mathrm{mm})$ represent the mean $\pm \mathrm{SD}$ (standard deviation)

${ }^{1} \mathrm{SScT}_{0}$ : Normal tendon; ${ }^{2} \mathrm{SScT}_{\mathrm{F}}$ : Full-thickness tear $<70 \%$ of tendon attachment; ${ }^{3} \mathrm{SScT}_{\mathrm{C}}$ : Near-complete tear having a full thickness tear $\geq 70 \%$ of tendon attachment.

*Differences in CHD between groups were analyzed with the ANOVA. Two-tailed $p$ value less than 0.05 was considered statistically significant.

Lesions of the long head of the biceps tendon were observed in $39(50.6 \%)$ of 77 shoulders in the patient groups. In the SScTC group, lesions of the biceps tendon were observed in all shoulders, including 2 unstable tendons and 4 complete tears (Table 2). Of all the biceps tendon lesions, 70 (76.1\%) were associated with a subscapularis tendon tear, and that finding was statistically

significant $(\mathrm{p}<0.001)$. Decentering of the humeral head $(1 / 56$ normal SScT vs. 11/34 SScT tear) was significantly associated with a SScT tear $(p=0.002)$. Abnormalities in the LT of the humeral head were occasionally observed in the patient groups, but there was no correlation between the LT changes and the presence of a SScT tear $(\mathrm{p}=0.329)$.

Table 2: Assessment of MR findings that could be related to subcoracoid stenosis.

\begin{tabular}{|c|c|c|c|c|c|c|}
\hline & & Control & ${ }_{1} \mathrm{SScT}_{0}$ & ${ }^{2} \mathbf{S S c T}_{\mathrm{F}}$ & ${ }^{3} \mathrm{SScT}_{\mathrm{C}}$ & $p$ value \\
\hline \multirow{2}{*}{ Subcoracoid bursa } & No & 25 & 18 & 12 & 3 & \multirow{2}{*}{0.115} \\
\hline & presence & 0 & 14 & 26 & 4 & \\
\hline \multirow{2}{*}{ SLAP lesion } & No & 17 & 15 & 22 & 5 & \multirow{2}{*}{0.581} \\
\hline & Presence & 8 & 17 & 16 & 2 & \\
\hline \multirow{4}{*}{ LHBT abnormality } & Normal & 25 & 24 & 14 & 0 & \multirow{4}{*}{$<0.001^{*}$} \\
\hline & Stable LHBT & 0 & 8 & 8 & 1 & \\
\hline & Unstable LHBT & 0 & 0 & 12 & 2 & \\
\hline & Complete tear & 0 & 0 & 4 & 4 & \\
\hline \multirow{3}{*}{$\begin{array}{l}\text { Lester tuberosity } \\
\text { change }\end{array}$} & No & 25 & 30 & 30 & 6 & 0.329 \\
\hline & BM edema & 0 & 1 & 5 & 0 & \\
\hline & Cystic change & 0 & 1 & 3 & 1 & \\
\hline \multirow{2}{*}{$\begin{array}{l}\text { Decentering of } \\
\text { humeral head }\end{array}$} & No & 25 & 31 & 30 & 4 & $0.002^{*}$ \\
\hline & presence & 0 & 1 & 8 & 3 & \\
\hline
\end{tabular}

Data represent the number of patients

1SScT0: Normal tendon; 2SScTF: Full-thickness tear $<70 \%$ of tendon attachment; 3SScTC: Near-complete tear having a full thickness tear $\geq 70 \%$ of tendon attachment.

*Two-tailed $\mathrm{p}$ value less than 0.05 was considered statistically significant.

\section{Discussion}

The present study proposed a new method to determine the minimal coracohumeral distance (CHD) using MPR MR image.
We hypothesized that a CHD obtained with the new MPR method (CHD3D) could more accurately reflect subcoracoid stenosis than traditional methods that measure the minimal distance on an MR axial image (CHDax), because the CHD3D could make it possible to 
measure the true shortest distance between the coracoid process and the humeral head in the 3-dimensional space. The CHD3D was well correlated with the CHDax. Furthermore, the CHD3D was significantly smaller in a near-complete tear of the SScT, suggesting a significant relationship between subcoracoid stenosis and SScT abnormalities, but the CHDax failed to show this association. It is fairly obvious that the CHDax which is measured in one certain axial MR image in various humeral head rotations could not represent the real shortest distance between the coracoid process and the lesser tuberosity.

Subcoracoid impingement is known to be an uncommon cause of chronic anterior shoulder pain $[1,3-5,7,12,13,15]$. It is usually diagnosed with characteristic clinical symptoms and a physical examination and is frequently overlooked in routine practice if there is no clinical suspicion. Although several investigators have tried to establish the cut-off value of the CHD to help diagnose subcoracoid impingement, the role of imaging in diagnosing subcoracoid impingement is still controversial [2,4,8-10,12,13,22]. Some authors have found that the CHD was between $8.6 \sim 11 \mathrm{~mm}$ in healthy subjects $[2,4,8,10,12,13,23]$ and a coracohumeral space of less than $6 \mathrm{~mm}$ [10] or $10 \mathrm{~mm}$ [14] was considered indicative of subcoracoid stenosis in previous studies. However, other authors thought that subcoracoid impingement could not be adequately assessed with the CHD on imaging. Giaroli et al. [4] said that if they adopted a cut-off value of a $5.5 \mathrm{~mm}$ coracohumeral interval for the diagnosis of subcoracoid impingement as reported by Friedman et al. [2], they could achieve a sensitivity value of only $5.3 \%$, but a specificity of $97.6 \%$. Hekimoğlu et al. [9] also concluded that with the ROC curve analysis, a proper cut-off value with sensitivity and specificity ranges higher than $80-90 \%$ could not be obtained for all average coracohumeral intervals [9]. Previous studies found that the CHD could be related to subcoracoid impingement but was poorly predictive of this diagnosis when acquired via a routine MRI $[13,4,9,14,8]$.

The CHD is likely to be a poor predictor of subcoracoid impingement because of the limitations of static MRI and fixed shoulder positioning during MR scan. The CHD is known to be narrowest during forward flexion and internal rotation of the shoulder $[2,23,24]$. However, the patient's arm is usually placed beside the supine patient and the shoulder is in a neutral or externally rotated to visualize the rotator cuff tendons during routine MR imaging. Although dynamic imaging is needed to obtain the true narrowest distance and to evaluate the subcoracoid soft tissues in the provoked position, it is dependent on patient cooperation and is potentially limited by pain $[13,14]$. Friedman et al. attempted a cine magnetic resonance imaging of the subcoracoid region in 1998, but it is not widely used today [2]. Furthermore, 2-D MR imaging could be limited in the assessment of the CHD in the 3-D space. 2-D MR images are obtained on fixed imaging planes, so the CHD measured on a 2-D MR image is only a projection of the 3-D CHD. These problems were overcome by using a 3D MR imaging and reconstruction technique.

Recent technical advancements of MR protocols and computer systems have allowed the generation of high quality postprocessing multiplanar reformats (MPR) from static MR imaging instead of dynamic MR imaging. In our study, the 3-D center of rotation of the humeral head in the glenohumeral joint (3-D COR) was determined, and two MPR images were then generated to obtain the shortest distance between the 3-D COR of the humeral head and the lesser tuberosity of the humeral head or coracoid process. By calculating the difference between these two measurements, we could obtain the shortest CHD, just as it could be obtained in the forward flexion and internal rotation position. Although the postprocessing steps to obtain the MPR images are somewhat time-consuming and inconvenient, the new method could be the best way to measure the true narrowest CHD in static MR imaging. The distance $(\sim 4 \mathrm{~mm})$ was smaller than that measured on axial MR images $(\sim 9 \mathrm{~mm})$. In contrast to CHDax, which demonstrated no correlation with the severity of the SScT abnormality, the CHD3D was smaller in patients with a near-complete tear of the SScT compared to other patient and control groups. This shows that the CHD3D is feasibly a better predictor of subcoracoid impingement than the CHDax.

In our study, all shoulders in the patient groups had fullthickness tears of the supraspinatus tendon, but there was no difference in the CHD (either CHDax or CHD3D) between the control and patient groups, which is consistent with recent studies by Bergin et al. [13] and MacMahon et al. [14]. Their reports demonstrated that the SScT was significantly more likely to be abnormal if the supraspinatus was retracted than if no retraction was present. The CHD decreased in the presence of a full-thickness tear of the supraspinatus tendon but there was no statistical significance $[13,14]$. As in previous results, LHBT abnormalities were frequently associated with tears of the SScT. Decentering of the humeral head was also more frequently observed in the patient group with near-complete tears of the SScT because subluxation of the humerus could potentially narrow the coracohumeral distance, predisposing the patient to subcoracoid impingement and, thus, to subscapularis lesions [14]. No significant differences were found in the frequency of bony changes in the lesser tuberosity, which is known to be associated with subcoracoid impingement.

There were several limitations in our study. First, we cannot evaluate the feasibility of CHD3D for the diagnosis of subcoracoid impingement because we could not select a clearly defined group of patients with subcoracoid impingement syndrome from our database. Subcoracoid impingement seems to be a largely clinical diagnosis supported, but not established, by MRI. We could not retrospectively diagnose impingement in our patient groups because careful physical examinations were not performed and there was no surgical correlation. As this is a retrospective study, it was impossible to obtain such clinical findings. Second, this is a retrospective cross-sectional study. At this point, we cannot be sure whether the narrowing of the CHD is a cause or result of a SScT tear. To date, we could not confirm that subcoracoid stenosis is a cause of a SScT tear or of subcoracoid impingement. Nevertheless, this study shows a significant relationship between a narrow CHD3D and a near-complete tear of the SScT. Third, a small number of patients in the patient group with near full-thickness tears of the SScT were analyzed. However, this is a result of the infrequent prevalence of 
this abnormality. Fourth, patients included in the control group were referred for MRI with the suspicion of other shoulder-related problems such as labral or glenohumeral ligament tears, bursitis, and adhesive capsulitis. Therefore, it was possible that any of those diseases could be another contributor to CHD narrowing. However, our results showed that there was no difference in the CHD between the control and patient groups with a tear in the supraspinatus tendon but an intact SScT (SScT0). Fifth, the methodology of how the measurements were performed was somewhat subjective because the shape of the humeral head and glenoid might influence the determination of the 3-D RC of the humeral head and the measurement of distances. However, our study demonstrated that two musculoskeletal radiologists could achieve perfect agreement in the measurement. Finally, our control and patient groups were not matched for age, sex or body mass index.

In conclusion, the shortest CHD could be reliably measured on 3-dimensional MPR MR images using the new method. The CHD3D was smaller in those with $a \geq 70 \%$ full-thickness tear of the SScT compared to those without. There was a greater statistical correlation for SScT abnormalities with the CHD3D than with the traditional CHDax. Our results showed that the CHD3D might be a reliable and useful method to predict subscapularis tendon abnormality. Future study should focus on validation of this new method to predict subcoracoid stenosis.

\section{Ethical Approval}

a) All procedures performed in studies involving human participants were in accordance with the ethical standards of the institutional and/or national research committee and with the 1964 Helsinki declaration and its later amendments or comparable ethical standards.

b) This retrospective study was approved by the institutional review board, and the need for informed consent was waived.

\section{References}

1. Drakes S, Thomas S, Kim S, Guerrero L, Lee SW (2015) Ultrasonography of subcoracoid bursal impingement syndrome. PM R 7(3): 329-333.

2. Friedman RJ, Bonutti PM, Genez B (1998) Cine magnetic resonance imaging of the subcoracoid region. Orthopedics 21(5): 545-548.

3. Garofalo R, Conti M, Massazza G, Cesari E, Vinci E, et al. (2011) Subcoracoid impingement syndrome: a painful shoulder condition related to different pathologic factors. Musculoskelet Surg 95(1): S2529.

4. Giaroli EL, Major NM, Lemley DE, Lee J (2006) Coracohumeral interval imaging in subcoracoid impingement syndrome on MRI. AJR Am J Roentgenol 186(1): 242-246.

5. Osti L, Soldati F, Del Buono A, Massari L (2013) Subcoracoid impingement and subscapularis tendon: Is there any truth? Muscles Ligaments Tendons J 3(2): 101-105.

6. Patte D (1990) The subcoracoid impingement. Clin Orthop Relat Res 254: 55-59.
7. Russo R, Togo F (1991) The subcoracoid impingement syndrome: Clinical, semeiologic and therapeutic considerations. Ital J Orthop Traumatol 17(3): 351-358.

8. Tracy MR, Trella TA, Nazarian LN, Tuohy CJ, Williams GR (2010) Sonography of the coracohumeral interval: A potential technique for diagnosing coracoid impingement. J Ultrasound Med 29(3): 337-341.

9. Hekimoglu B, Aydin H, Kizilgoz V, Tatar IG, Ersan O (2013) Quantitative measurement of humero-acromial, humero-coracoid, and coracoclavicular intervals for the diagnosis of subacromial and subcoracoid impingement of shoulder joint. Clin Imaging 37(2): 201-210.

10. Lo IK, Parten PM, Burkhart SS (2003) Combined subcoracoid and subacromial impingement in association with anterosuperior rotator cuff tears: An arthroscopic approach. Arthroscopy19(10): 1068-1078.

11. Misirlioglu M, Aydin A, Yildiz V, Dostbil A, Kilic M, et al. (2012) Prevalence of the association of subacromial impingement with subcoracoid impingement and their clinical effects. J Int Med Res 40(2): 810-815.

12. Richards DP, Burkhart SS, Campbell SE (2005) Relation between narrowed coracohumeral distance and subscapularis tears. Arthroscopy 21(10): 1223-1228.

13. Bergin D, Parker L, Zoga A, Morrison W (2006) Abnormalities on MRI of the subscapularis tendon in the presence of a full-thickness supraspinatus tendon tear. AJR Am J Roentgenol 186(2): 454-459.

14. Mac Mahon PJ, Taylor DH, Duke D, Brennan DD, O’Brien J, et al. (2007) Contribution of full-thickness supraspinatus tendon tears to acquired subcoracoid impingement. Clin Radiol 62(6): 556-563.

15. Graichen H, Bonel H, Stammberger T, Englmeier KH, Reiser M, et al. (1999) Subacromial space width changes during abduction and rotation-a 3-D MR imaging study. Surg Radiol Anat 21(1): 59-64.

16. Graichen H, Bonel H, Stammberger T, Haubner M, Rohrer H, et al. (1999) Three-dimensional analysis of the width of the subacromial space in healthy subjects and patients with impingement syndrome. AJR Am J Roentgenol 172(4): 1081-1086.

17. Lyons RP, Green A (2005) Subscapularis tendon tears. J Am Acad Orthop Surg 13(5): 353-363.

18. Yoo JC, Rhee YG, Shin SJ, Park YB, McGarry MH, et al. (2015) Subscapularis tendon tear classification based on 3-dimensional anatomic footprint: A cadaveric and prospective clinical observational study. Arthroscopy 31(1): 19-28.

19. Bland JM, Altman DG (1986) Statistical methods for assessing agreement between two methods of clinical measurement. Lancet 1(8476): 307310 .

20. Lee KM, Lee J, Chung CY, Ahn S, Sung KH, et al. (2012) Pitfalls and important issues in testing reliability using intraclass correlation coefficients in orthopedic research. Clin Orthop Surg 4(2): 149-155.

21. Lin LI (1989) A concordance correlation coefficient to evaluate reproducibility. Biometrics 45(1): 255-268.

22. Gerber C, Terrier F, Zehnder R, Ganz R (1987) The subcoracoid space. An anatomic study. Clin Orthop Relat Res 215: 132-138.

23. Gerber C, Terrier F, Ganz R (1985) The role of the coracoid process in the chronic impingement syndrome. J Bone Joint Surg Br 67(5): 703-708.

24. Ferreira Neto AA, Almeida AM, Maiorino R, Zoppi Filho A, Benegas E (2006) An anatomical study of the subcoracoid space. Clinics (Sao Paulo) 61(5): 467-472. 


\section{ISSN: 2574-1241}

DOI: 10.26717/BJSTR.2018.09.001875

Sae Hoon Kim. Biomed J Sci \& Tech Res

(C) (i) This work is licensed under Creative

Submission Link: https://biomedres.us/submit-manuscript.php

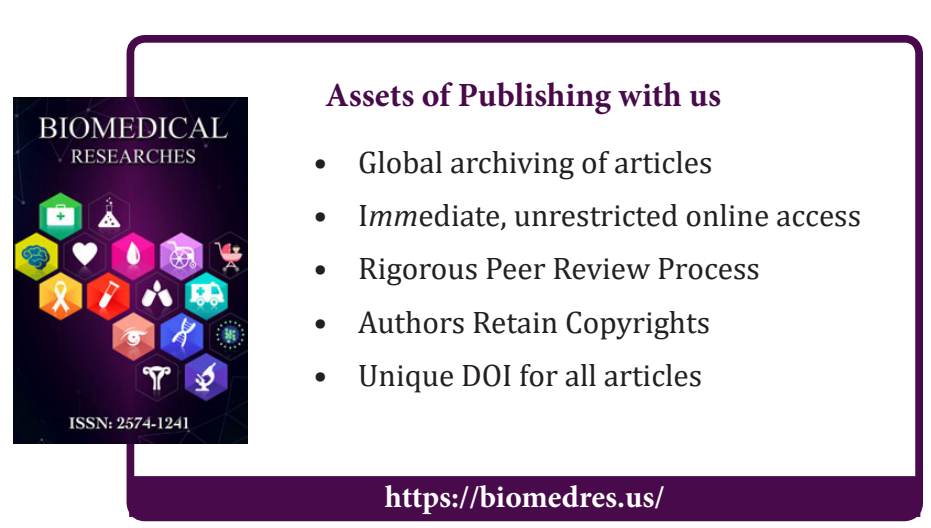

